\title{
Acute hospital at home - an integrated, holistic and multidisciplinary service
}

\author{
Authors: Will Divall, James Richards, Patricia McCormack, Sue Trim and Katie Whitlock
}

\section{Aims}

To assess the impact, effectiveness and ongoing development of an acute hospital at home (AH@H) service, created in January 2014.

\section{Methods}

Retrospective review of AH@H patients between January 2014 and September 2017 specifically focusing on patient satisfaction, inpatient hospital bed days saved, main conditions treated and re-admission rates. Of discharge to assess (D2A) patients between April 2016 and September 2017 focusing on bed days saved, reduction of care needs and regained independence.

\section{Results}

AH@H is a virtual inpatient ward run by a multidisciplinary team, who provide aspects of inpatient care to people in their own homes. Services provided include:

> intravenous antibiotics (including administration via Surefuser pump)

$>$ facility to insert and manage PICC/mid-lines.

$>$ consultant-led daily virtual ward round

$>$ twice weekly review by senior house officer doctor at home

$>$ nursing assessments and observations (recorded electronically at the patient's home, through VitalPac)

$>$ complex wound care, including VAC pump dressings

$>$ blood tests

> physiotherapy, occupational therapy, speech and language therapy and dietitian input

> coordination with ambulatory care to arrange specialist reviews, further tests or imaging.

Since 2016 a D2A service has been developed that bridges care packages while giving multidisciplinary therapy input.

The AH@H service treats patients with a variety of medical and surgical conditions, the most common are:

> respiratory infections- exacerbations of bronchiectasis/COPD/ asthma and pneumonia (17\%)
$>$ cellulitis $(14 \%)$

$>$ venous thromboembolism $(5 \%)$

$>$ septic arthritis ( $4 \%)$

$>$ infected diabetic foot ulcers $(4 \%)$.

Between January 2014 and September 2017, 1,433 patients have been managed through the AH@H service, saving on average 9.64 bed days per day, with a re-admission rate of only $4.75 \%$. A key success is the high degree of patient satisfaction: $98.13 \%$ of patients were 'likely or extremely likely to recommend the service' in the Friends and Family Test, compared with $83 \%$ on an inpatient ward. Patients feel they recover much better in their own home, eating their normal diet and being able to rest in a more relaxing environment without the disturbances of a busy inpatient ward. The average cost per day of an $\mathrm{AH} @ \mathrm{H}$ is $£ 100$, compared with $€ 280$ for an inpatient ward bed.

Between April 2016 and September 2017, D2A has managed 379 patients, saving on average 5.58 hospital bed days per day, while reducing $28.5 \%$ of patient's care needs and $21.11 \%$ regaining independence, no longer requiring a package of care.

\section{Conclusion}

AH@H has been a success and the service has grown with the addition of D2A and increased junior medical cover. The key successes.

$>$ An average of 15.22 bed days saved per day.

$>$ High patient satisfaction $-98.13 \%$.

> On average saving £180 per patient per day, compared with inpatient care.

$>49.6 \%$ of D2A patients regaining independence and having reduced care needs.

> Being adaptable to a variety of medical and surgical conditions to assist patient flow through the hospital.

\section{Conflict of interest statement}

None

Author: Dorset County Hospital Foundation Trust, Dorchester, United Kingdom 